\title{
Modified bisection method for solving nonlinear equations
}

\author{
Younbae Jun*, Junho Jeon \\ Department of Applied Mathematics, Kumoh National Institute of Technology, Gyeongbuk 39177, Korea \\ *Corresponding Author: Younbae Jun, Department of Applied Mathematics, Kumoh National Institute \\ of Technology, Gyeongbuk 39177, Korea
}

\begin{abstract}
This method is one of the numerical methods that is an extension of bisection method to solve nonlinear equations. The paper deals with the convergence and number of iterations with visual graph.
\end{abstract}

Keywords: Root-finding problem, bisection method, nonlinear equation.

\section{INTRODUCTION}

The root finding problem is a fundamental problem of numerical analysis. Express $x$ as a root satisfying the given equation $f(x)=0$. In numerical analysis, one of the main purposes is to develop a numerical algorithm to find approximate values. Well-known root finding methods [1,2] in numerical analysis are Newton's method, secant method, fixed point iteration method, and bisection method. In Newton method, given initial value $p_{0}$, a sequence $\left\{p_{n}\right\}_{n=1}^{\infty}$ is generated by $p_{n}=p_{n-1}-\frac{f\left(p_{n-1}\right)}{f^{\prime}\left(p_{n-1}\right)}$, for $n \geq 1$, which converges to a root. Since the derivatives are sometimes difficult to be obtained, the secant method in which the average rate of change is applied may be used instead of the derivatives in Newton method. Also fixed point iteration method is root finding method of $f(x)=0$ using the form of $g(x)=x$, in which a sequence is generated so that it converges to a root. The bisection method $[3,4,5,6,7]$ is one of root finding method. Cut half of the interval and use the Intermediate Value Theorem [8] to select the small sub-interval, where the root is present. Bisection method uses just two sub-intervals, but more than two sub-intervals can be set. In other words, the Intermediate Value Theorem is used for three or more sub-intervals. The contents of this approach are explained in Section 2. Furthermore, we deal with convergence and error bound. As well as bisection method, it is possible to estimate the number of iterations which will be visualized in a graph.

\section{Modified Bisection METHOD}

The bisection method is one of the methods of finding the root of the equation $f(x)=0$. Suppose $f$ is a continuous function defined on the interval $[a, b]$, with $f(a)$ and $f(b)$ of opposite sign. This method cuts half of the interval $[a, b]$ and selects a sub-interval satisfying the Intermediate Value Theorem. Initialize the selected sub-interval again at both ends, and then cut the half again. Repeatedly running this procedure yields the error bound as small as the interval of the last iteration.

Figure 1 shows the first three steps of bisection method, in which $p_{3}$ is obtained in the third iteration with the error bound of $\frac{b_{3}-a_{3}}{2}$. Therefore, if the bisection method is repetitively run as shown in Figure 1 , then approximate value is convergent to a root.

Bisection method uses the mid-point as the approximate value. If we think differently, we can see that it consists of two equally spaced sub-intervals. For example, if we define $h$ as $\frac{b-a}{2}$ in the interval $[a, b]$, the two small sub-intervals are defined as follows: $[a, a+h],[a+h, b]$ 


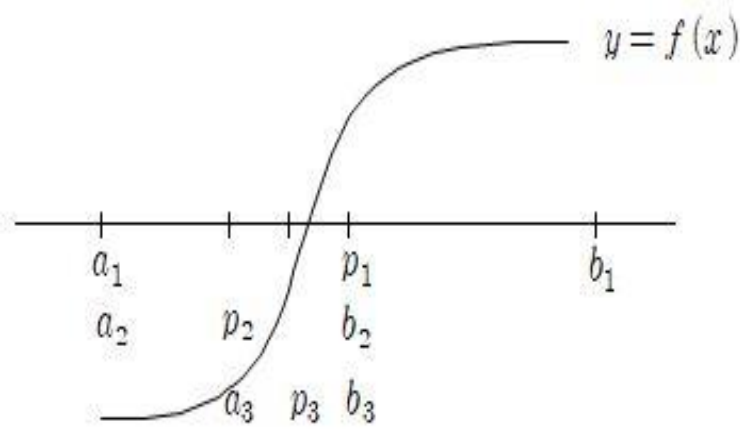

Figure1. Diagram of the bisection method

In the bisection method, since the middle value is approximation, likewise we use $a+h$ as the approximate value. Then, if the interval $[a, b]$ is composed of three equally spaced sub-intervals with the increment $h=\frac{b-a}{3}$, we get $[a, a+h],[a+h, a+2 h],[a+2 h, b]$

If Intermediate Value Theorem is applied for each sub-interval, it is possible to know whether a root exists in the sub-interval. Then, choose the interval in which the root is present and define it again as $a$ and $b$, so that new $h$ is further redefined. The Intermediate Value Theorem can then be used for each sub-interval. Then, the error is guaranteed by the length of the sub-interval in the last iteration. In this process, we can get a better approximation.

It is very important to know the convergence in root finding problem. Because if approximation does not converge at a particular value, you cannot be sure that it is really meaningful as a value. Therefore, we prove convergence and an error bound for the method, so that the approximation converges to a true value when the number of iterations becomes sufficiently large.

Theorem 2.1 Suppose that $f$ is a continuous function on $[a, b]$ and $f(a) f(b)<0$. Assume the interval is divided into equally spaced $s$ sub-intervals. Then the sequence $\left\{p_{n}\right\}_{n=1}^{\infty}$ approximates a zero $p$ of $f$ with $\left|p_{n}-p\right| \leq \frac{b-a}{s^{n}}$.

Proof. Let $a_{1}=a, b_{1}=b$ and set $h_{n}=\frac{b_{n}-a_{n}}{s}$ for each $n \geq 1$. We can pick approximations $p_{n_{1}}=$ $a_{n}+h_{n}, p_{n_{2}}=a_{n}+2 h_{n}, \cdots, p_{n_{k}}=a_{n}+k h_{n}$. Since $k=s-1$,

$$
p_{n_{k}}=a_{n}+k h_{n}=a_{n}+k\left(\frac{b_{n}-a_{n}}{s}\right)=a_{n}+(s-1)\left(\frac{b_{n}-a_{n}}{s}\right)=\frac{1}{s} a_{n}+\left(1-\frac{1}{s}\right) b_{n}
$$

Also since $a_{n} \leq p \leq b_{n}, a_{n}-\left(\frac{1}{s} a_{n}+\frac{s-1}{s} b_{n}\right) \leq p-p_{n_{k}} \leq b_{n}-\left(\frac{1}{s} a_{n}+\frac{s-1}{s} b_{n}\right)$

$$
0 \leq\left(1-\frac{1}{s}\right)\left(b_{n}-a_{n}\right) \leq p-p_{n_{k}} \leq \frac{1}{s}\left(b_{n}-a_{n}\right)
$$

Because $b_{n}-a_{n}=\frac{b_{n-1}-a_{n-1}}{s}=\cdots=\frac{b_{1}-a_{1}}{s^{n-1}}$, we have

$0 \leq p-p_{n_{k}} \leq \frac{b_{1}-a_{1}}{s^{n}}$, for $n \in N$.

Hence $p_{n_{k}} \rightarrow p$ as $n \rightarrow \infty$, that is $p_{n} \rightarrow p$ as $n \rightarrow \infty$.

We try to estimate the number of iterations using the error bound. For given error bound $\epsilon$, the number of iterations $n$ satisfies the following inequality:

$\frac{b-a}{s^{n}} \leq \epsilon$, that is, $n \geq \frac{\ln \left(\frac{b-a}{\epsilon}\right)}{\ln s}$

Since $a, b, \epsilon, s$ are all constants in the inequality above, the number of iteration can be predicted. For the sake of simplicity, we use $n \geq \frac{\ln c}{\ln s}$, where $c=\frac{b-a}{\epsilon}$. 


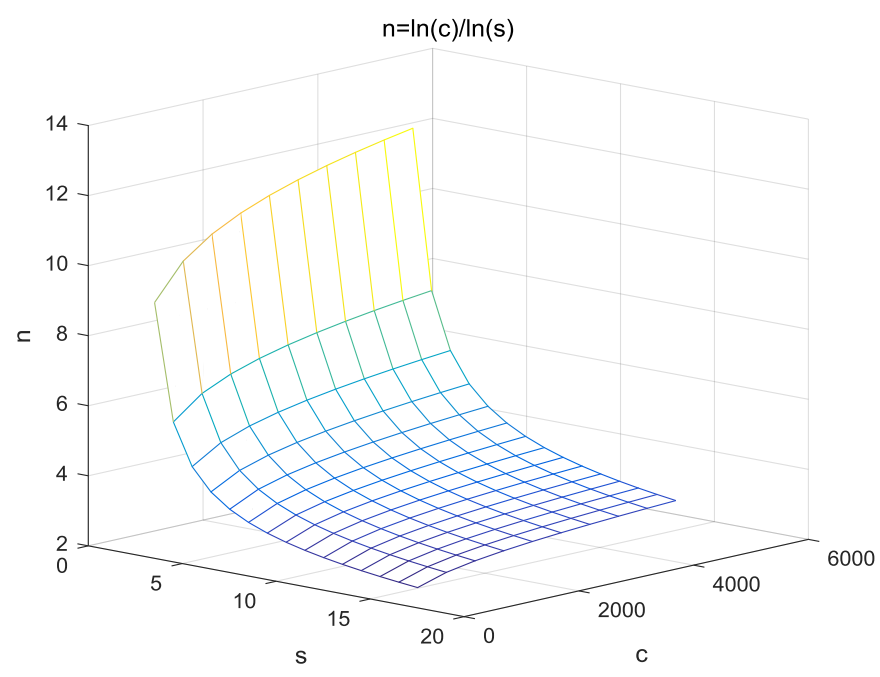

Figure2. Graph of the relation $n=\frac{\ln c}{\ln s}$ for various $c$ and $s$

Figure 2 shows the graph of the number of iterations for the various error bound and the number of subintervals. In Figure 2, we see that the number of iterations decreases as the number of sub-intervals increases. For example, it can be seen from the graph that $n$ decreases as $s$ increases at $c=2000$. And when $s$ is a constant, $n$ increases as the $c$ increases.

Example1. Solve the non-linear equation $3 x-e^{x}=0$ using the modified bisection method with three sub-intervals. Note that the exact solution is $x=1.5121345516578424739 \cdots$.

\begin{tabular}{|c|c|c|}
\hline$n$ & Bisection method & Modified bisection method \\
\hline 1 & 1.500000 & 1.500000 \\
\hline 2 & 1.750000 & 1.500000 \\
\hline 3 & 1.625000 & 1.500000 \\
\hline 4 & 1.562500 & 1.500000 \\
\hline 5 & 1.531250 & 1.512346 \\
\hline 6 & 1.515625 & 1.512346 \\
\hline 7 & 1.507813 & 1.512346 \\
\hline 8 & 1.51179 & 1.512346 \\
\hline 9 & 1.513672 & 1.512193 \\
\hline 10 & 1.512695 & 1.512142 \\
\hline 11 & 1.512207 & 1.512142 \\
\hline 12 & 1.511963 & 1.512137 \\
\hline 13 & 1.512085 & $\mathbf{1 . 5 1 2 1 3 5}$ \\
\hline 14 & 1.512146 & \\
\hline 15 & 1.512115 & \\
\hline 16 & 1.512131 & \\
\hline 17 & 1.512138 & \\
\hline 18 & $\mathbf{1 . 5 1 2 1 3 5}$ & \\
\hline
\end{tabular}

In the table above, we see that the modified bisection method converges to the actual root faster than the bisection method.

\section{CONCLUSION}

In this paper, we presented the modified bisection method to solve nonlinear equations. We estimated the number of iterations and provided a visual graph of them for the various error bounds and the number of sub-intervals. Unlike the Newton's method or the fixed point method, the new method is not affected by the function. That is, no special conditions are required. Only the existence of the solution is enough. So it can be used for many kinds of functions. 


\section{REFERENCES}

[1] M. Basto, T. Abreu, V. Semiao, and F.L. Calheiros, Convergence and dynamics of structurally identical root finding methods, Appl. Numer. Math. 120 (2017), 257-269.

[2] A.A. Krushynska, Root finding method for problems of elastodynamics. Comput. Assist. Mech. Eng. Sci. 17 (2010), 3-11.

[3] G.E. Collins, On the maximum computing time of the bisection method for real root isolation, J. Symbolic Comput. 79 (2017), 444-456.

[4] R.H. Goodman, J.K. Wróbel, High-order bisection method for computing invariant manifolds of twodimensional maps. Internat. J. Bifur. Chaos Appl. Sci. Engrg. 21 (2011), 2017-2042.

[5] C. Gutierrez, F. Gutierrez and M-C. Rivara, Complexity of the bisection method, Theoret. Comput. Sci. 382 (2007), 131-138.

[6] A. Jahanshahloo, M. Zohrehbandian, Using bisection method for solving linear bilevel programming problems. Adv. Appl. Math. Sci. 15 (2016), 195-204.

[7] A. Malyshev, M. Sadkane, A bisection method for measuring the distance of a quadratic matrix polynomial to the quadratic matrix polynomials that are singular on the unit circle. BIT 54 (2014), 189-200.

[8] R.L. Burden, J.D. Faires, and A.M. Burden, Numerical Analysis, Cengage, 2018.

Citation: Younbae Jun \& Junho Jeon (2019). Modified bisection method for solving nonlinear equations. International Journal of Scientific and Innovative Mathematical Research (IJSIMR), 7(9), pp. 8-11. http://dx.doi.org/ 10.20431/2347 -3142.0709003

Copyright: () 2019 Authors, this is an open-access article distributed under the terms of the Creative Commons Attribution License, which permits unrestricted use, distribution, and reproduction in any medium, provided the original author and source are credited. 